\title{
TÍNH TOÁN VÀ MÔ PHỎNG HIỆN TƯợNG MA SÁT TRƯợT ĐỐI VỚI VÂT LĂN ỨNG DUNG TRONG THIẾT KẾ HÊ THỐNG CẤP - THOÁT PHÔI CỦ๋ MÁY IN LỤA BÁN TỰ ĐộnG KIỂU MỚI
}

\author{
VÕ THÀNH KIỆT, ĐẶNG HOÀNG MINH \\ Khoa Công nghệ Co khí, Truờng Đại học Công nghiệp Thành phố Hồ Chi Minh \\ danghoangminh@iuh.edu.vn
}

Tóm tắt. Bài báo trình bày việc tính toán góc nghiêng cần thiết của một mặt phẳng nghiêng để đáp ứng yêu cầu về thời gian lăn (có trượt và không trượt) của vật hình trụ và ứng dụng trong việc thiết kế bộ phận cấp - thoát phôi của máy in lụa bán tự động kiểu mới. Những công thức tìm được chỉ đúng với giả thiết các vật lăn là đồng chất và hình dạng trụ. Còn với các vật lăn có hình dạng phức tạp với nhiều thành phần vật liệu khác nhau (nắp chai, thân chai), tác giả đã sử dụng phần mềm Recurdyn để mô phỏng và tìm ra góc nghiêng cần thiết. Kết quả mô phỏng đã cho thấy, sai số giữa công thức ở trường hợp lý tưởng và phần mềm mô phỏng ở khoảng trên - dưới 10\%, từ đó có thể thiết kế được các bộ phận cấp - thoát phôi của máy in lụa trong điều kiện thực tế. Cụ thể là máng cấp phôi có góc nghiêng là khoảng từ $0.14 \div 2.3^{\circ}$, máng thoát phôi cần có góc nghiêng từ $7 \div 10^{\circ}$ để đảm bảo yêu cầu về năng suất in. Bài báo là một ví dụ điển hình trong việc ứng dụng mô phỏng vào việc thiết kế các hệ thống cơ khí và máy móc ở Việt Nam.

Từ khoá. In lụa, cấp phôi tự động, ma sát trượt, ma sát lăn, mô phỏng động lực học, Recurdyn.

\section{CALCULATION AND SIMULATION OF SLIDING FRICTION WITH ROLLING OBJECT APPLIED IN THE DESIGN OF THE WORKPIECE FEED-EXIT SYSTEM OF THE NEW SEMI-AUTOMATIC SILK SCREEN PRINTER}

\begin{abstract}
The paper presents the calculation of the required inclination angle of an inclined plane to meet the rolling time requirements (with slip and no-slip) of a cylindrical object and its application in the design of the workpiece feed-escape parts of new semi-automatic silk screen printing machine. The formulas found are only true assuming the rolling bodies are homogeneous and cylindrical. As for the roller with complex shapes with many different material components (bottle cap, bottle body), Recurdyn software was used to numerically investigate the necessary angle of inclination. The simulation results show that the error between the formula in the ideal case and the simulation software is around $10 \%$, thus it is possible to design the feed - escape module of the screen printing machine in the actual event. Specifically, the inclined chute for the workpiece feeding has an angle about $0.14 \div 2.3^{\circ}$ of inclination, and the workpiece draining needs to have an angle of $7 \div 10^{\circ}$ to ensure printing productivity requirements. The paper is a typical example of the application of simulation to the design of mechanical systems and machines in Vietnam.
\end{abstract}

Keywords. Screen printing, automatic loading, sliding friction, roller friction, dynamic simulation, Recurdyn.

\section{GIỚI THIỆU}

Xã hội ngày càng phát triển thì nhu cầu in lụa trong đời sống hiện đại là rất lớn. Ví dụ như để quảng bá về thương hiệu và tạo danh tiếng cho một công ty, tập đoàn, cơ sở kinh doanh hoặc một tổ chức nào đó sẽ cần in hình ảnh logo, khẩu hiệu thương mại hoặc thông tin liên hệ của mình trên các sản phẩm như quần áo, bút viết, dây đeo thẻ, cốc nhựa hoặc thủy tinh, v.v... Với những nhu cầu đó, năm 1962 Edels và Kensington đã chế tạo máy in lụa với cơ cấu dùng băng tải để làm dây chuyền vận chuyển chi tiết in thành một quy trình khép kín, có buồng sấy nhiệt để mực khô nhanh sau khi in tránh tình trạng mực bị nhòe [1]. Cùng khoảng thời gian này Louis Giuhert Dubult đã phát minh một chiếc máy in lụa với dạng chi tiết in dạng hình côn [2].

Năm 1965 Fiegel trong luận văn [3] của mình đã nghiên cứu sự hình thành và phát triển của ngành in lụa, sau đó miêu tả một quy trình in lụa hoàn toàn đơn giản. Ba năm sau đó (1968), trong sáng chế của mình, Peter Zimmer đã tạo ra một chiếc máy in lụa hoàn toàn mới [4] với khung in có dạng hình trụ, răng cưa ngoại vi, ổ trục dọc, ... Mặc dù những thành thành tựu nghiên cứu trong lĩnh vực chế tạo máy in lụa ngày 

THIÊT KẾ HỆ THỐNG CÂP - THOÁT PHÔI CỦA MÁY IN LỤA BÁN TỬ ĐỘNG KIỂU MỚI

nay là tương đối lớn, nhưng đi kèm với những ưu điểm của chúng là một loạt các vấn đề tồn tại vẫn chưa được khắc phục triệt để. Điển hình như ở dạng máy in lụa cho cơ sở sản xuất vừa và nhỏ hiện nay thì thiết kế nhỏ gọn là một yêu cầu quan trọng của máy, giúp tiết kiệm không gian. Đặc biệt là ở nước ta hiện nay, hầu hết các xưởng in lụa vẫn còn đang là các cơ sở sản xuất vừa và nhỏ. Thông qua khảo sát thực tế tại một số xưởng in lụa với quy mô vừa và nhỏ trên địa bàn $\mathrm{Tp} . \mathrm{HCM}$, tác giả nhận thấy công tác in lụa hiện nay vẫn được thực hiện một cách thủ công là chủ yếu $[5,8]$, quá trình in ấn phụ thuộc vào tay nghể của người thợ, các loại máy in lụa thường là những loại máy cơ đơn giản với năng suất thấp [5-9], cường độ công việc nặng nhọc. Còn trên thị trường, các loại máy in lụa tự động của nước ngoài thường có giá thành khá cao và chỉ thích hợp với quá trình sản xuất công nghiệp, sản xuất hàng loạt, in ấn chỉ với một loại sản phẩm là chủ yếu [10-13]. Xuất phát từ những vấn đề như trên, nhóm sinh viên Đại học Công Nghiệp TP.HCM [5] đã đưa ra ý tưởng và thiết kế, chế tạo thử nghiệm một máy in lụa bán tự động kiểu mới (Hình 1) với năng suất cao, có thể in được đa dạng các loại sản phẩm dạng tròn xoay. Công tác hiệu chỉnh được đơn giản hóa để một người công nhân không lành nghề vẫn có thể dễ dàng điều chỉnh khung in hay gá đặt sản phẩm. Ngoài ra việc chế tạo đơn giản sẽ làm giảm giá thành của máy để phù hợp hơn với khả năng tài chính của các xưởng in lụa vừa và nhỏ tại Việt Nam.

Đối với máy in lụa được đề cập ở trên, thì vấn đề cấp và thoát phôi là một bài toán quan trọng. Việc sử dụng các dây chuyền cấp và thoát phôi tự động đòi hỏi cần có thêm các thiểt bị như động cơ, băng chuyền, các thiết bị điều khiển. Do đặc thù vật in là các chi tiết hình trụ, nên việc cấp - thoát phôi có thể ứng dụng nguyên lý vật tự lăn trên mặt phẳng nghiêng. Nguyên lý này dựa trên hiện tượng lăn có ma sát của vật phẳng và chuyển động song phẳng của vật rắn [14-18].

Yêu cầu đặt ra trong việc thiết kế bộ phận cấp - thoát phôi dựa trên hiện tượng này là cần thiết kế góc nghiêng của bề mặt lăn, cũng như độ dài của bề mặt nghiêng để thời gian lăn của phôi từ vị trí cấp đến vị trí chờ in, cũng như từ vị trí in (khi in xong) đến vị trí chờ lấy hàng đáp ứng yêu cầu về mặt năng suất, khớp với thời gian thao tác in của máy [19]. Nếu như góc nghiêng càng lớn thì thời gian vật lăn càng nhanh, tuy nhiên nếu vượt qua một ngưỡng nào đó thì vật sẽ chuyển sang trạng thái vừa lăn vừa trượt, điều này sẽ gây nên xây xát bề mặt vật in, hay hoặc tốc độ nhanh quá sẽ làm các vật bị va chạm mạnh vào nhau, khiến cho vật bị bập bênh, mất ổn định khi rơi vào vị trí chờ.

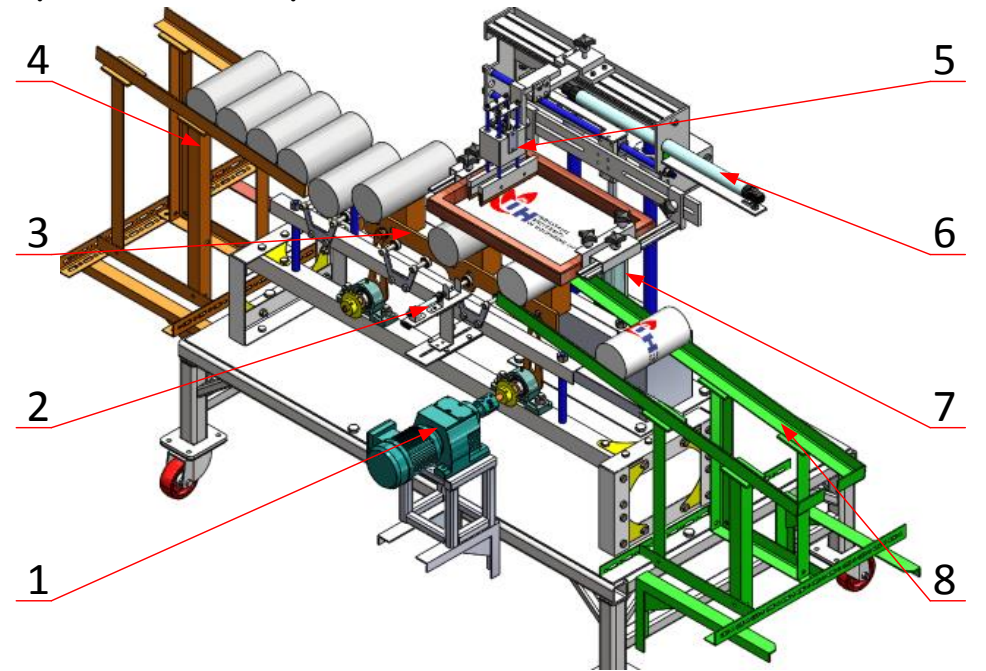

Hình 1: Thiết kế máy in lụa kiểu mới

1. Động cơ

2. Xylanh định vị chi tiết in

3. Cụm cơ cấu nâng phôi

4. Máng cấp phôi

5. Cụm cơ cấu dao in/phủ mực

6. Xylanh dẫn hướng khung lụa

7. Xylanh nâng hạ khung in

8. Máng thoát phôi 
Việc tính toán và mô phỏng vật lăn trên mặt phẳng nghiêng về lý thuyết không phải là một bài toán mới [14-15, 20-21]. Tuy nhiên hiện tượng này chia ra làm 2 trường hợp: Lăn không trượt và lăn có trượt. Khi góc nghiêng nhỏ (máng cấp phôi) thì vật có xu thế lăn không trượt [17-18, 21]. Ngược lại ở máng thoát phôi, do nhu cầu thoát phôi nhanh nên góc nghiêng có thể lớn, khi đó cần xét cả hiện tượng vật vừa lăn vừa trượt [16]. Đa số các công bố về cơ học cổ điển [14-15, 20-21] chỉ xét đến hiện tượng lăn không trượt. Chỉ có số ít tài liệu [16-18] xét về hiện tượng vừa lăn vừa trượt. Tuy nhiên việc xây dựng một công thức tổng hợp, nói lên sự chuyển đổi trạng thái giữa hai hiện tượng khi góc nghiêng thay đổi thì chưa tìm được ở các công trình này. Vì vậy trong bài báo này tác giả đã xây dựng công thức cho cả hai hiện tượng. Mặc dù vậy, trong điều kiện làm việc thực tế, các vật phẩm cần in có các độ cứng khác nhau, vật liệu khác nhau, hình dáng đa dạng, điều này ảnh hưởng đến hiện tượng tiếp xúc giữa bề mặt vật phẩm và máng. Trên thực tế khi quan sát các vật trụ lăn ta thường thấy vật có một độ nẩy nhất định. Điều này là do độ cứng của vật liệu và độ nhám bề mặt. Những yếu tố này chưa được xét tới trong các hệ thức giải tích. Do đó cần mô phỏng trên các phần mềm đa vật lý. Tuy nhiên, hiện nay ở nước ta, việc thiết kế các máy móc và hệ thống cơ khí thường được thực hiện dựa trên kinh nghiệm và phương pháp thử - sai. Việc tính toán và phân tích các hiện tượng vật lý phức tạp xảy ra trong các máy móc cần thiết kế thường được thực hiện một cách rất hạn chế do đa số các kỹ sư chưa nắm được các quy trình tính toán và mô phỏng một cách chuyên nghiệp. Trong khi đó việc mô phỏng lại có thể giúp cho các nhà thiết kế có cái nhìn rõ nét để đánh giá một cách gần đúng, tính khả thi trong các phương án thiết kế, từ đó tránh được các quá trình thực nghiệm thử - sai, tiết kiệm được nhiều chi phí về mặt thời gian và tính kinh tế [22]. Các công bố về hiện tượng ma sát của vật lăn là rất hạn chế. Phần nhiều là nghiên cứu các hiện tượng ma sát trượt [24-25]. Trên thực tế hiện tượng ma sát của vật lăn phức tạp hơn ma sát trượt nhiều ở các yếu tố như sự chuyển tiếp giữa trượt và lăn, sự biển dạng, vật liệu giữa các bề mặt, v.v... Việc lựa chọn các thông số mô phỏng hợp lý để khảo sát hiện tượng ma sát của vật lăn và đưa ra các thiết kế máy móc phù hợp hiện nay chưa được nghiên cứu một cách đầy đủ.

Vì vậy, trong bài báo này các tác giả sẽ nghiên cứu mô phỏng hiện tượng này trên phần mềm Recurdyn [22-23]. Quá trình mô phỏng được thực hiện dựa trên nguyên tắc bổ sung từng yếu tố để đánh giá mức độ ảnh hưởng của các thông số hiện tượng. Đầu tiên là mô phỏng trong tình trạng lý tưởng là vật cứng tuyệt đối để so sánh với công thức giải tích. Nếu sai số giữa kết quả mô phỏng và công thức tính toán giải tích lớn hơn $10 \%$, thì lúc này cần bổ sung thêm các yếu tố như độ cứng, hệ số giảm chấn, ngưỡng vận tốc tĩnh và ngưỡng vận tốc động vào trong phần mềm Recurdyn để tăng độ chính xác khi mô phỏng. Từ đó xác định được bộ tham số mô phỏng phù hợp với thực tế.

\section{CƠ SỞ LÝ THUYẾT}

Bộ phận cấp và thoát phôi của máy in lụa kiểu mới làm việc dựa trên nguyên lý vật trụ tròn lăn trên mặt phẳng nghiêng. Vì các sản phẩm đang xét phải có dạng hình trụ (Hình 2):

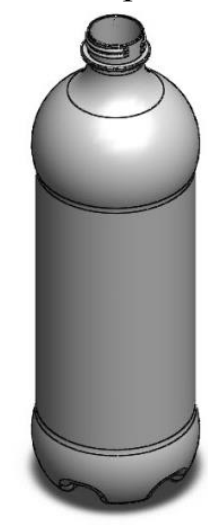

(a)

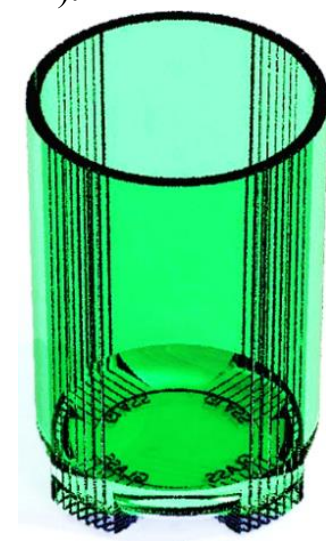

(b)

Hình 2:Các vật trụ tròn cần in lụa (a)-Chai, (b)-Cốc

Bài toán đặt ra phải thiết kế được góc nghiêng của máng trượt để sao cho các sản phẩm lăn thoả mãn được yêu cầu về mặt thời gian, năng suất. Để làm được bài toán này thì ta xét vật lăn trên mặt phẳng nghiêng với các yếu tố như Hình 3. 


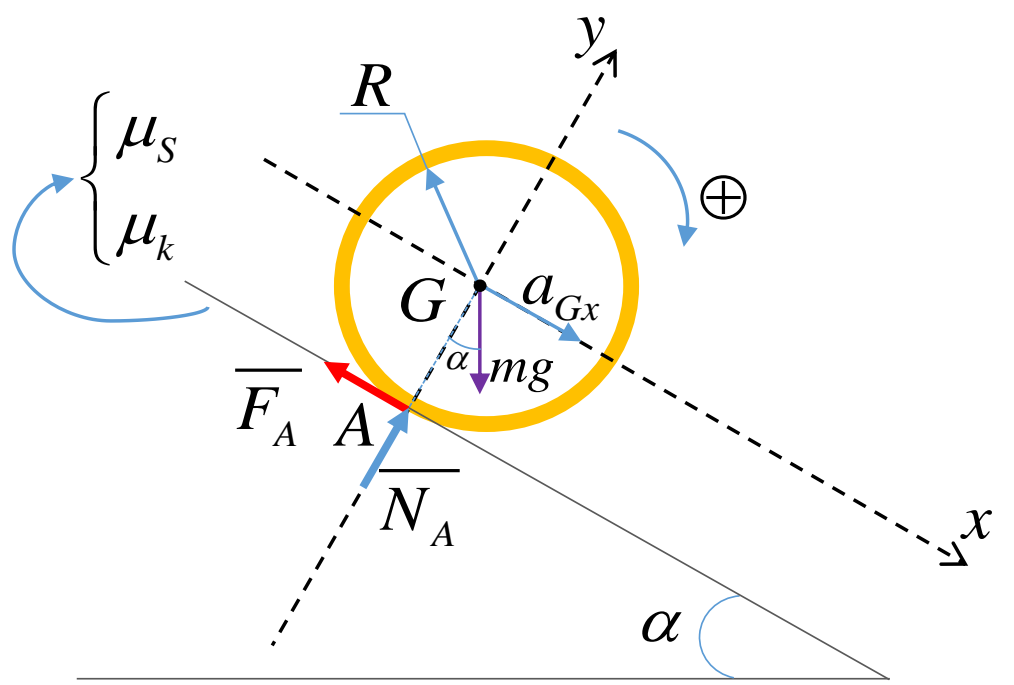

Trong đó:

$-\mu_{S}$ : Hệ số ma sát tĩnh

$-\mu_{k}$ : Hệ số ma sát động

- $F_{A}$ : Lực cản vật trượt

- $N_{A}$ : Phản lực tại diểm $A$

- $R$ : Bán kính của chai

- $g$ : Gia tốc trọng trường

- $m$ : Khối lượng của vật

- $a_{G x}$ : Gia tốccủa vật theo trục $x$

- $\alpha$ : Góc nghiêng của mặt phẳng

Hình 3: Xét vật trên mặt phẳng nghiêng

Hệ phương trình động lực học mô phỏng chuyển động của vật [21] có dạng:

$$
\left\{\begin{array}{l}
\sum F_{k x}=m \cdot a_{G x} \Leftrightarrow-F_{A}+m g \sin \alpha=m \cdot a_{G x} \\
\sum F_{k y}=m \cdot a_{G y} \Leftrightarrow N_{A}-m g \cos \alpha=0 \\
\sum M_{G}=I_{G z} \cdot \varepsilon_{z} \Leftrightarrow F_{A} \cdot R=I_{G z} \cdot \varepsilon_{z}
\end{array}\right.
$$

Với: $a_{G x}$ : Gia tốc tại điểm $G$ xét theo trục $x ; a_{G y}$ : Gia tốc tại điểm $G$ xét theo trục $y$;

$I_{G z}$ : Moment quán tính tại điểm $G$ theo trục $z ; \varepsilon_{z}$ : Gia tốc góc

Dựa vào hệ phương trình (1) và các hệ thức động học chúng ta xây dựng được các hệ thức xác định thời gian lăn của vật như sau:

Thời gian vật di chuyển đến chân dốc:

$$
t_{S}=\left\{\begin{array}{l}
\sqrt{\frac{2 S\left(m R^{2}+I_{G z}\right)}{m g R^{2} \cdot \sin \alpha}} \text { nếu } \\
\sqrt{\frac{4 S}{g\left(2 \sin \alpha-2 \mu_{k} \cos \alpha\right)}} \\
\alpha>\arctan \left(\left(1+\frac{m R^{2}}{I_{G z}}\right) \mu_{S}\right)
\end{array}\right.
$$

Đối với vật lăn có dạng là ống trụ tròn mỏng (tương ứng với các dạng chai, lọ cần in) thì ta có $I_{G z}=m R^{2}$, từ đó ta có:

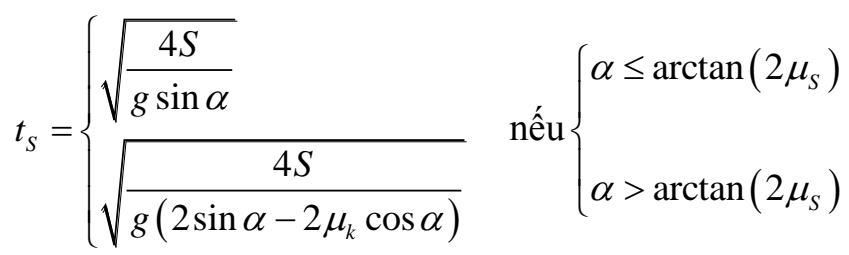

Công thức (3) cho thấy đối với trường hợp vật có dạng ống trụ thành mỏng lý tưởng lăn không trượt thì thời gian di chuyển của vật sẽ chỉ phụ thuộc vào góc nghiêng và quãng đường đi chuyển, tức là không phụ thuộc vào vật liệu của vật lăn. Còn trong trường hợp vật vừa lăn vừa trượt thì thời gian di chuyển của vật sẽ còn phụ thuộc thêm vào hệ số ma sát động, tức là có phụ thuộc vào vật liệu của vật lăn. Công thức (3) cho phép xác định được góc nghiêng $\alpha$ dựa vào thời gian $t_{S}$ cần thiết.

\section{QUY TRÌNH THIẾT KẾ VỚI PHẦN MỀM MÔ PHỎNG RECURDYN}

Trong thực tế, để thực hiện các thí nghiệm nghiên cứu thì cần rất nhiều các trang thiết bị kèm theo, do đó chi phí cho mỗi vấn đề nghiên cứu sẽ rất tốn kém. Để khắc phục vấn đề này, các nhà nghiên cứu đã làm ra nhiều phần mềm với chức năng hỗ trợ mô phỏng các thí nghiệm khác nhau, điển hình trong số đó là phần 
mềm Recurdyn [23]. Với các tính năng mô phỏng động lực học mạnh mẽ, kèm theo sự chính xác cao, Recurdyn đã trở thành một trong những phần mềm đáng để sử dụng cho việc mô phỏng cũng như phân tích các bài toán nghiên cứu [22].

Để kiểm nghiệm được độ chính xác cũng như tiết kiệm được thời gian thiết kế mô hình thực nghiệm, tác giả đã sử dụng phần mềm Recurdyn để mô phỏng các chuyển động trong bộ phận cấp - thoát phôi cho máy in lụa bán tự động kiểu mới. Ngoài ra phần mềm còn giúp cho tác giả kiểm nghiệm lại sự chuẩn xác của các công thức đã được xây dựng ở trên. Để có thể thực hiện được bài toán này, tác giả đã tuân theo quy trình thực hiện như sau (Hình 4):

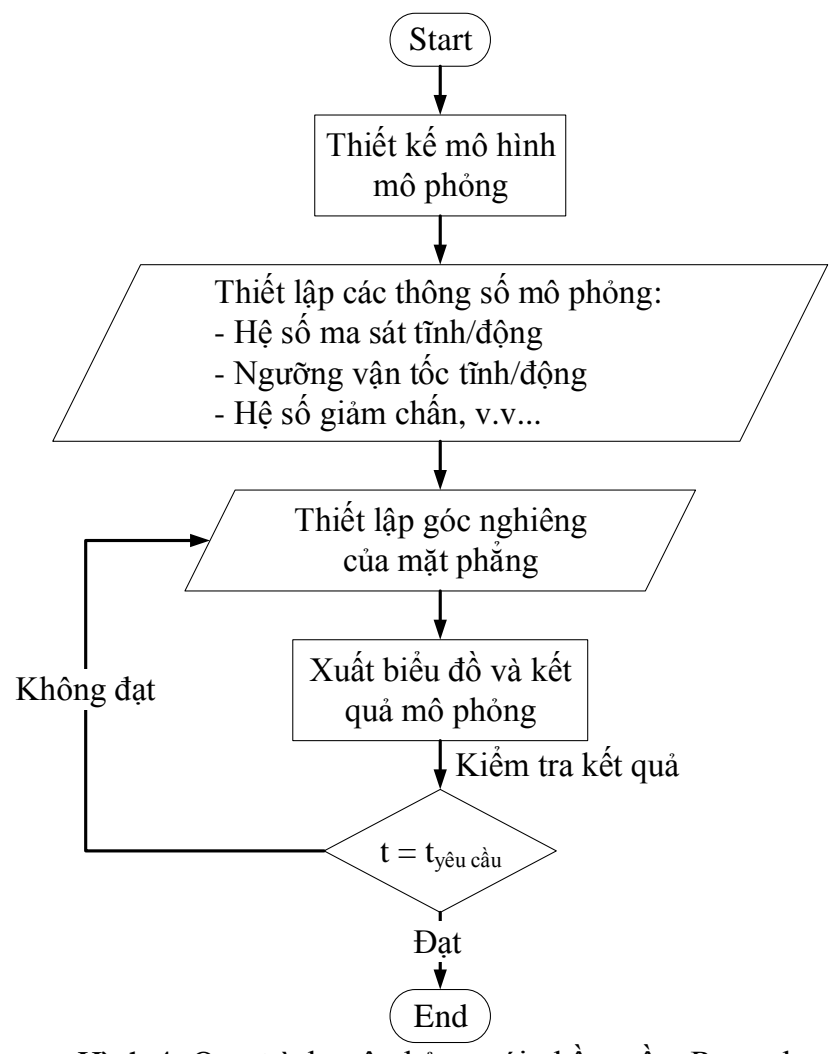

Hình trụ lý tường
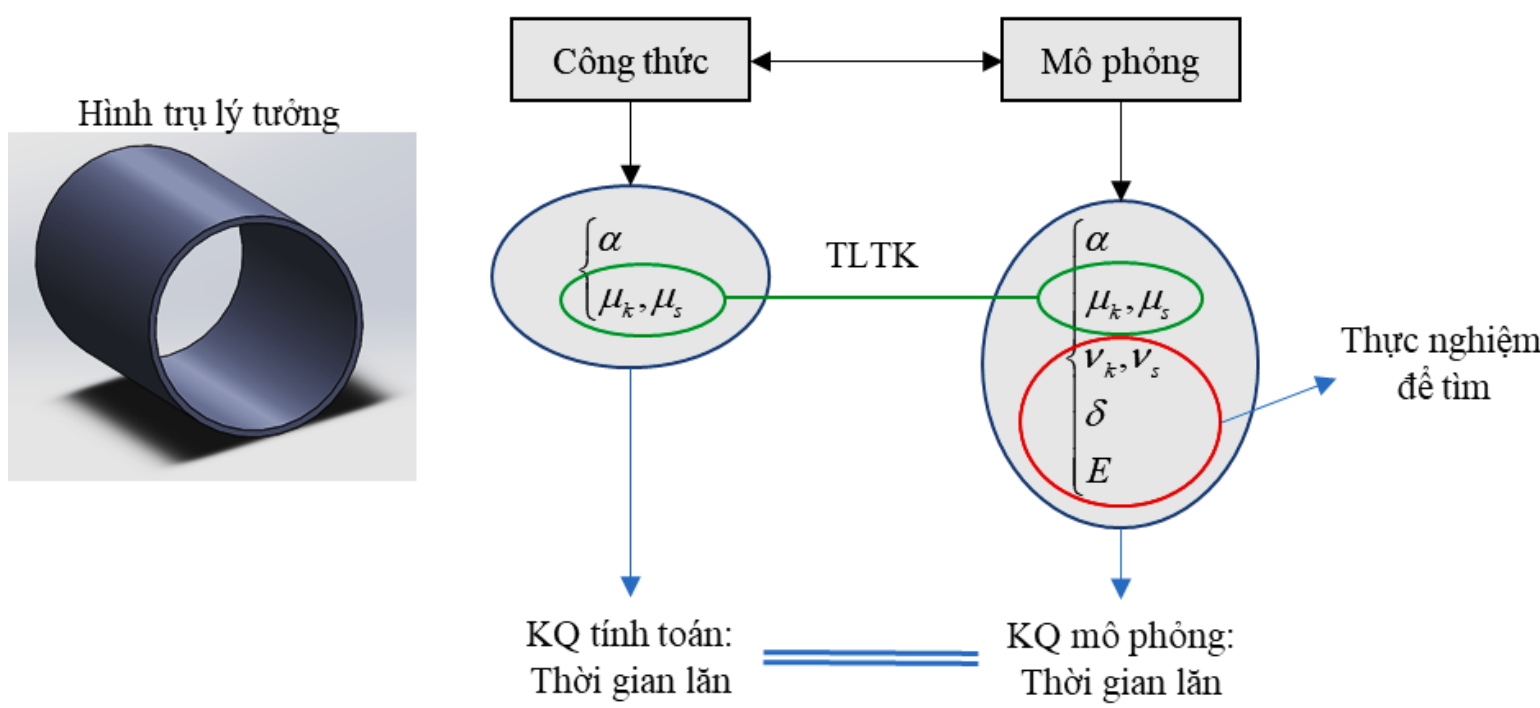

Hình 5: Bài toán xuôi: Xác định các thông số mô phỏng 
Trong đó:

$\mu_{S}$ : Hệ số ma sát tĩnh; $\mu_{k}$ : Hệ số ma sát động; $v_{s}$ : Ngưỡng vận tốc tĩnh; $v_{d}$ : Ngưỡng vận tốc động

$\delta$ : Hệ số giảm chấn; $E$ : Độ cứng của vật;

Vòng tròn màu xanh lá cây: Các giá trị lấy từ tài liệu tham khảo

Vòng tròn màu đỏ: Các giá trị dựa vào thực nghiệm để xác định

Mô hình mô phỏng có thể được thiết kế trực tiếp trên phần mềm Recurdyn với các vật thể đơn giản hoặc các phần mềm $3 \mathrm{D}$ thông dụng như Solidworks, $\mathrm{NX}$, v.v... rồi chuyển tiếp vào phần mềm Recurdyn.

Với phần mềm mô phỏng Recurdyn thì có rất nhiều hệ số liên quan đến các hiện tượng ma sát của vật lăn mà chúng ta chưa có thông tin từ ban đầu. Những hệ số này liên quan đến nhiều hượng tượng vật lý phức tạp hơn, xoay quanh bài toán ma sát chính. Để thiết kế các bộ phận máy in lụa thì những hiện tượng này không nhất thiết cần xét tới. Tuy nhiên, giá trị của những hệ số này cần phải được xác định để sao cho kết quả của việc mô phỏng là đáng tin cậy. Phương pháp xác định như sau: Bằng việc khảo sát hiện tượng lăn của một vật hình trụ mỏng đơn giản, đồng chất với nhiều góc alpha cho trước, và một vài dạng vật liệu cho trước (nhựa và nhôm). Dựa vào tài liệu tham khảo (TLTK) [17-18, 22-23] chúng ta xác định hệ số ma sát tĩnh và động $\left(\mu_{S}, \mu_{k}\right.$ : trong vòng tròn hình xanh lá cây của Hình 5$)$ và áp dụng cho cả công thức và phần mềm mô phỏng. Sau đó điều chỉnh các hệ số mô phỏng còn lại $\left(v_{s}, v_{d}, \delta, E\right.$ : trong vòng tròn màu đỏ) sao cho kết quả thời gian lăn của vật từ phần mềm mô phỏng và công thức tính toán là bằng nhau.

Bảng 1: Thông số mô phỏng tìm được

\begin{tabular}{|l|c|}
\hline Static Threshold Velocity (Ngưỡng ma sát tĩnh) & 1.0 \\
\hline Dynamic Threshold Velocity (Ngưỡng ma sát động) & 1.5 \\
\hline Static Friction Coefficient (Hệ số ma sát tĩnh) & $0.28 \div 0.35$ \\
\hline Dynamic Friction Coefficient (Hệ số ma sát động) & $0.33 \div 0.37$ \\
\hline Hệ số giảm chấn & $2 \div 5$ \\
\hline Độ cứng của vật & $24000 \div 25000$ \\
\hline
\end{tabular}

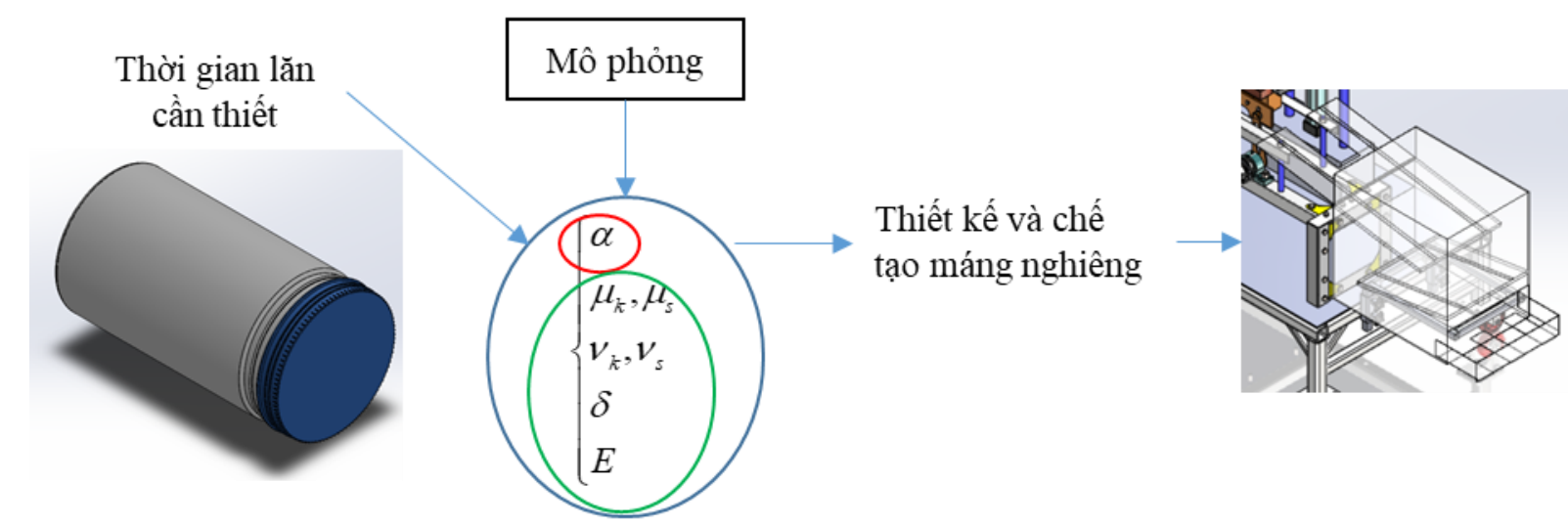

Hình 6: Bài toán ngược: Xác định góc nghiêng cần phải thiết kế đối với vật lăn có hình dạng phức tạp, không đồng chất

Trên cơ sở các hệ số $\left(\mu_{S}, \mu_{k}, v_{s}, v_{d}, \delta, E\right)$ đã được xác định ở bước trên (Bảng 1$)$, ta giải quyết bài toán thiết kế ngược. Xuất phát từ thời gian lăn cần thiết của vật in lụa, ta xác định góc nghiêng alpha cần thiết của máng nghiêng. Lúc này thì vật lăn là một vật có hình dạng phức tạp và không đồng chất, tuy nhiên thành phần của chúng chỉ là nhựa và nhôm đã được xem xét ở bước trên khi tìm ra các hệ số mô phỏng (Bảng 1). Từ đó ta thiết kế được máng nghiêng cho bộ phận cấp và thoát phôi (Hình 6).

Áp dụng các quy trình trên vào việc thiết kế máy in lụa. Thông qua phần mềm Solidworks thiết kế mẫu chai (Hình 7) và phần mềm Recurdyn mô phỏng, tác giả đã xây dựng được đồ thị (Hình 8) xác định thời gian di chuyển của vật phụ thuộc vào góc nghiêng $\alpha$. 


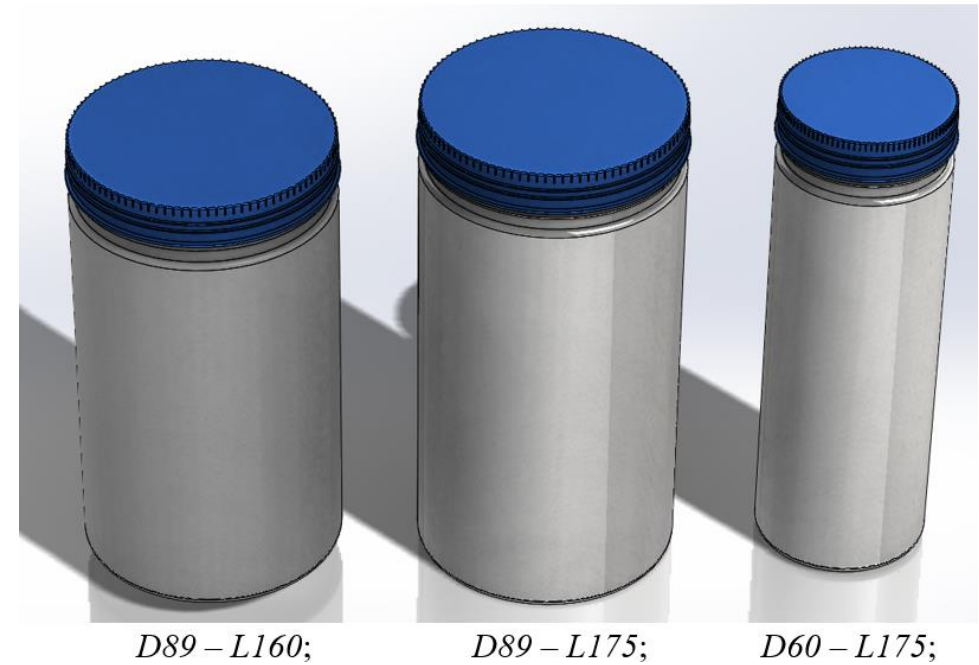

*Chú thích:

Hình 7: Mô hình các mẫu chai 3D sử dụng cho mô phỏng

D: Đường kính

$L:$ Chiều dài chai

$C T$ : Công thức- Kết quả được dựa theo số liệu tính toán của công thức số (3), bao gồm các đại lượng như quãng đường, gia tốc trọng trường và góc nghiêng.

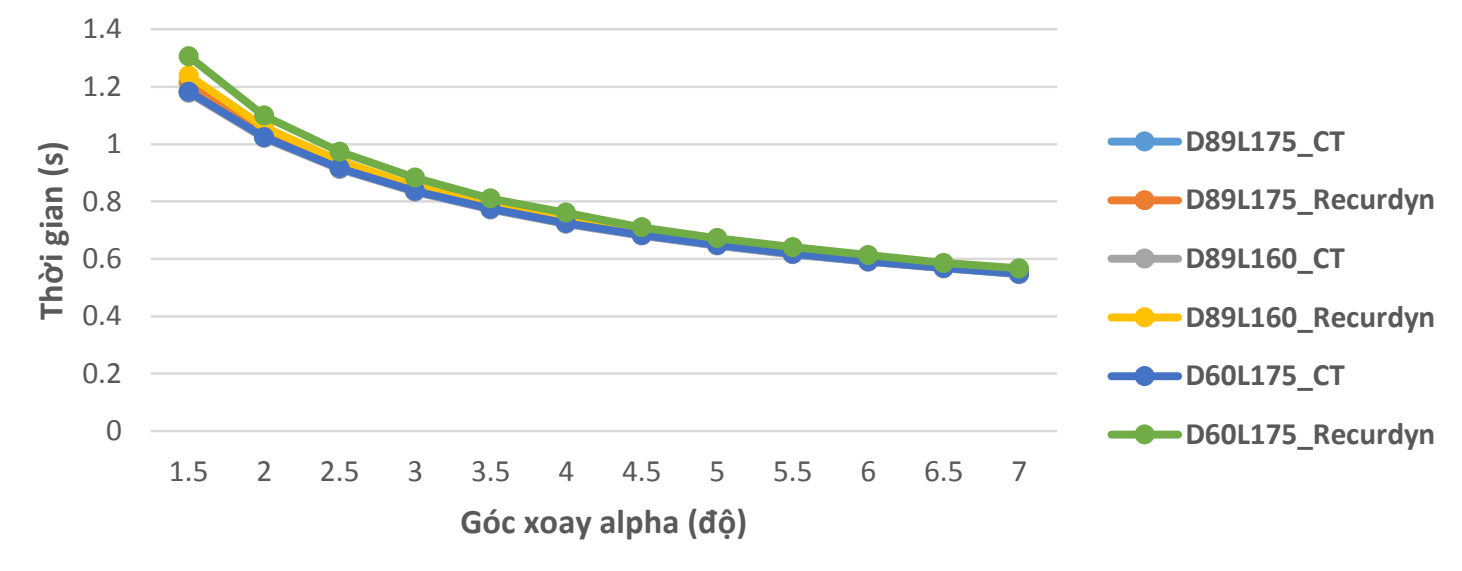

Hình 8: Biểu đồ thể hiện thời gian phụ thuộc góc alpha

Kiểm nghiệm mô phỏng với sản phẩm 3D:

Bảng 2: Sai số so sánh giữa các dạng mô phỏng

\begin{tabular}{|c|c|c|c|}
\hline STT & $\begin{array}{l}\text { Cách mô phỏng - Loại liên kết sử } \\
\text { dụng }\end{array}$ & Ý nghĩa & Sai số (\%) \\
\hline 1 & $\begin{array}{c}\text { Geo Surface - Surface(Shell, PatchSet), } \\
\text { Surface(Shell, PatchSet) }\end{array}$ & $\begin{array}{l}\text { Tạo mối liên kết va chạm giữa } 2 \text { vật thể } \\
\text { dựa trên biên dạng và bề mặt tiếp xúc }\end{array}$ & $1.49 \sim 10.47$ \\
\hline 2 & $\begin{array}{c}\text { Geo Surface - Solid(Shell, PatchSet), } \\
\text { Solid(Shell, PatchSet) }\end{array}$ & $\begin{array}{c}\text { Tạo mối liên kết va chạm giữa } 2 \text { vật thể } \\
\text { dựa trên độ cứng của chúng }\end{array}$ & $1.57 \sim 12.16$ \\
\hline
\end{tabular}

Sai số ở đây chính là sự chênh lệch giữa kết quả mô phỏng và kết quả tính theo công thức lý thuyết. Trong môi trường mô phỏng Recurdyn, phần mềm tính toán dựa trên nhiều tham số khác nhau, như độ cứng, độ giảm chấn hay khối lượng của các thành phần, ngoài ra còn có sự tác động của ngưỡng vận tốc tĩnh và ngưỡng vận tốc động, còn trong công thức (3) thì vẫn chưa xét đến các yếu tố đó. Do đó với các góc có độ 
nghiêng càng thấp, thì sự ảnh hưởng bởi các tham số càng nhiều, nên sẽ dẫn đến việc sai số càng lớn, và ngược lại khi độ nghiêng mặt phẳng càng lớn thì sai số sẽ càng ít đi.

\section{THIẾT KẾ VÀ CHẾ TẠO THỰC NGHIỆM}

Từ công thức (3) cũng như mô phỏng sẽ chọn được góc nghiêng hợp lý để cho thời gian lăn của vật kịp với tốc độ chuyển phôi, đáp ứng được với yêu cầu năng suất.

Các thông số kỹ thuật yêu cầu [5,7]:

Đường kính chai 3 loại: $60 \mathrm{~mm}=0.06 \mathrm{~m}, 89 \mathrm{~mm}=0.089 \mathrm{~m}$.

Chiều dài chai 2 loại: $160 \mathrm{~mm}=0.16 \mathrm{~m}, 175 \mathrm{~mm}=0.175 \mathrm{~m}$.

Khối lượng chai 3 loại: $25 \mathrm{~g}, 22 \mathrm{~g}, 19 \mathrm{~g}$

Quãng đường cấp phôi: $S=2 \times R=100 \mathrm{~mm}=0.1 \mathrm{~m}$

Quãng đường thoát phôi: $S=1 \mathrm{~m}$

Hệ số ma sát tĩnh: 0.35

Hệ số ma sát động: 0.3

Với những thông số như trên, ta thu được thời gian để hoàn thành 1 sản phẩm là $t=4.2$ giây (Hình 9).

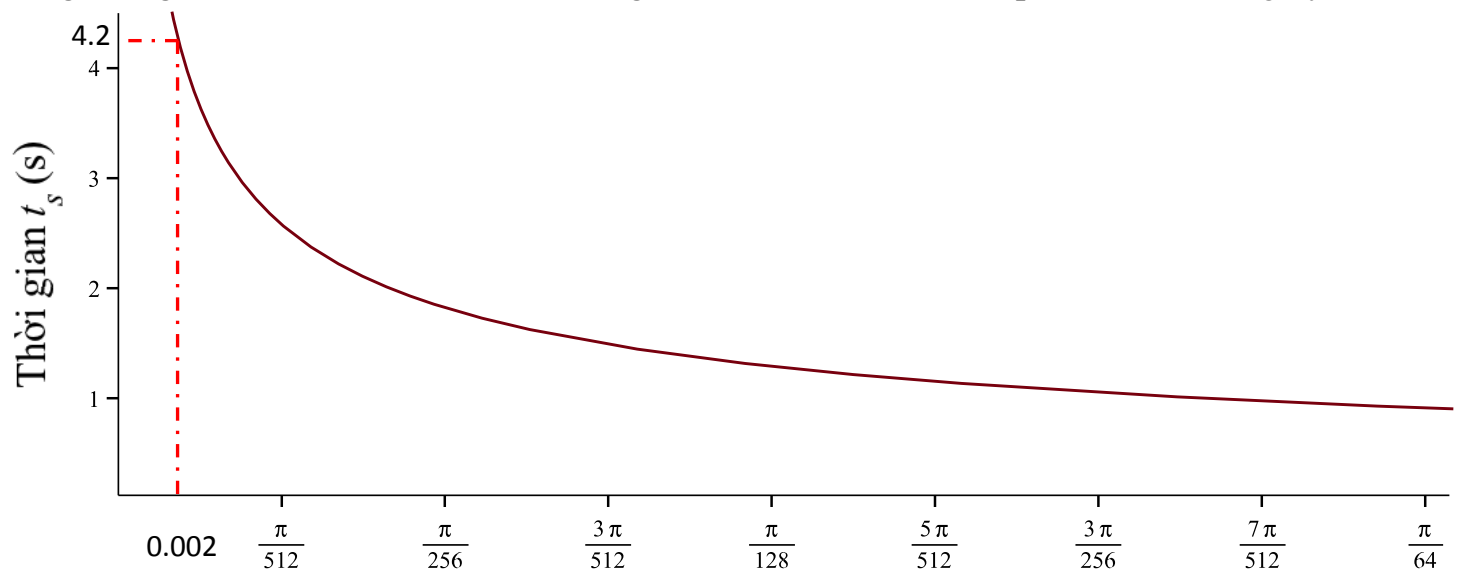

Góc nghiêng $\alpha(\mathrm{rad})$

\subsection{Thiết kế góc nghiêng cho bộ phận cấp phôi}

Hình 9: Biểu đồ thời gian vật lăn phụ thuộc góc nghiêng

Yêu cầu bài toán đặt ra cho năng suất của máy là cần in 850 sản phẩm trong vòng 1 giờ $[5,7]$. Từ thiết kế cơ cấu chuyển phôi có dạng hình bình hành của máy (Hình 11) ta tính được thời gian để chai từ vị trí hàng chờ tới vị trí chờ chuyển chai phải thấp hơn thời gian hoàn thành 1 sản phẩm, tức là $t_{S}<4.2$ giây và để đáp ứng được chuyện đó thì $\alpha_{1}>0.002 \mathrm{rad}$. Giả sử $t_{S}=1$ giây:

Theo công thức (3) thì ta sẽ thu được $\alpha_{1}=2.3^{\circ}$ (Hình 10). Như vậy góc nghiêng $\alpha_{1}$ đã thoả mãn yêu cầu về năng suất của bài toán.

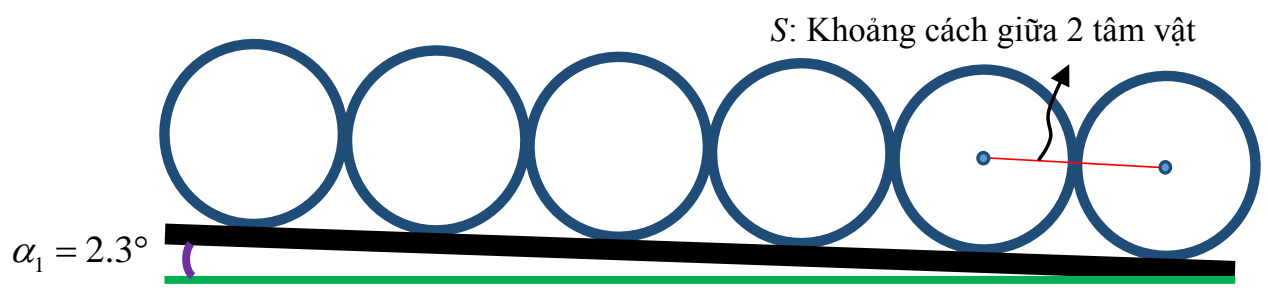

Hình 10: Góc nghiêng bộ phận cấp phôi

Để cho vật có thể lăn chậm thì người thiết kế cần phải chọn góc nghiêng thật bé, nhưng trong thực tế thì không thể như vậy được. Vì để có thể lắp ráp và căn chỉnh chính xác với góc nghiêng rất nhỏ thì sẽ rất khó, tốn nhiều thời gian và không thực sự cần thiết đối với nhu cầu của người sử dụng. Ngoài ra, khi chọn góc nghiêng quá cao thì vận tốc vật lăn sẽ lớn, điều này sẽ dẫn đến hiện tượng vật bị văng ra ngoài hoặc bị sai lệch vị trí in ngay khi tiếp xúc với khối đỡ chữ V (Hình 11).

Nguyên lý hoạt động của cơ chế chuyển phôi: Chi tiết in (2) từ máng trượt được đẩy vào vị trí 1 , sau đó cơ cấu bốn khâu bản lề (3) chuyển động quay đều để nâng chi tiết in đến vị trí 2 , cùng lúc này chi tiết tiếp theo từ máng trượt sẽ được đẩy vào vị trí 1 . Cơ cấu bốn khâu bản lề (3) chuyển động vòng tiếp theo và 
nâng chi tiết ở vị trí 1 , vị trí 2 sang vị trí 2 , vị trí 3 , lúc này khung in lụa (1) sẽ hạ xuống thực hiện quá trình in. Quá trình này được lặp đi lặp lại và tạo thành một quy trình khép kín.

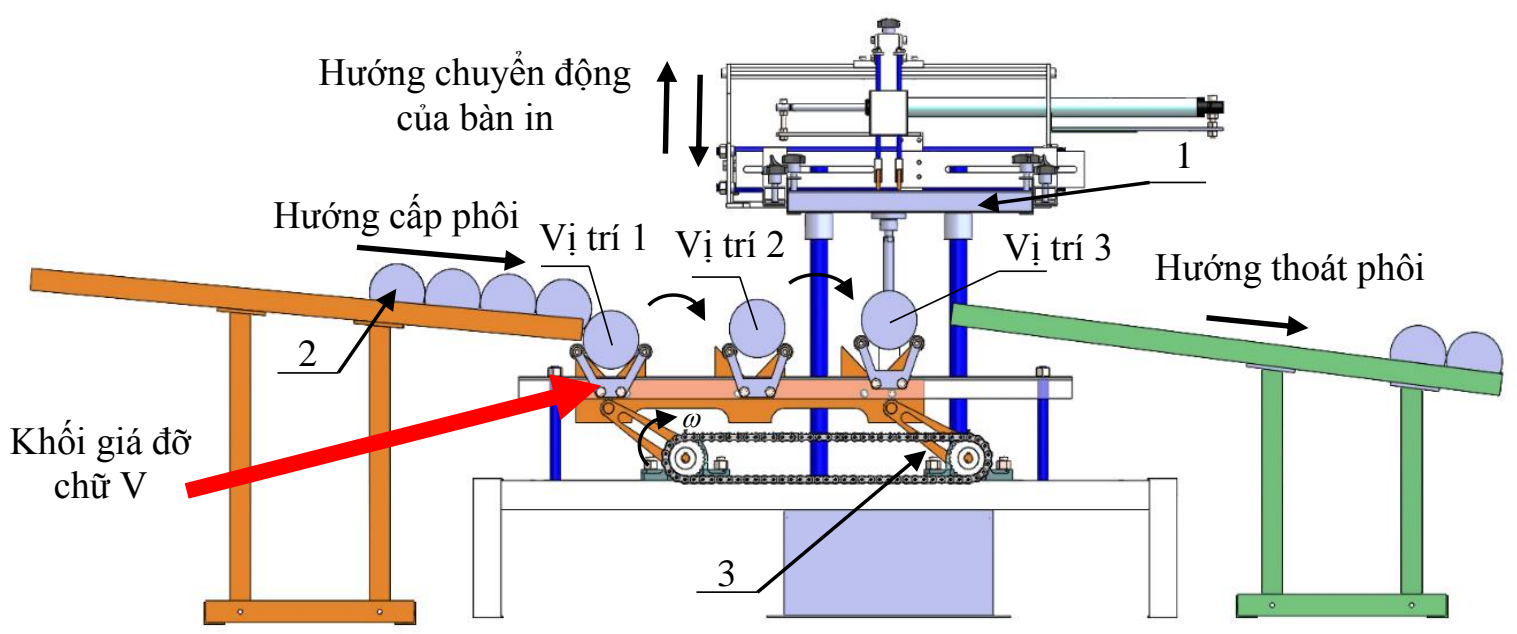

Hình 11: Góc nghiêng bộ phận thoát phôi

Dựa vào yếu tố trên, tác giả đã chọn góc nghiêng $\alpha_{1}$ dao động từ $0.14^{\circ}-2.3^{\circ}$ tức thời gian chậm nhất là 4.1 giây và nhanh nhất là 1 giây. Như vậy vật có thể lăn hết quãng đường trong quá trình cấp phôi vì nó đã thoả mãn được các yêu cầu đặt ra như:

$$
\begin{array}{ll}
\circ & t_{s}<4.2 \text { giây } \\
\circ & \text { Có thể dễ dàng lắp ráp và căn chỉnh } \\
\bigcirc & \text { Vận tốc vận lăn không quá nhanh }
\end{array}
$$

\subsection{Thiết kế góc nghiêng cho bộ phận thoát phôi}

Yêu cầu bài toán đặt ra cho thùng thoát phôi:

- Đảm bảo cho bề mặt chai sau khi in không tiếp xúc trực tiếp với mặt phẳng nghiêng của thùng thoát phôi.

- Thời gian thoát phôi nhanh, không bị va chạm với phôi tiếp theo $(t<4.2$ giây).

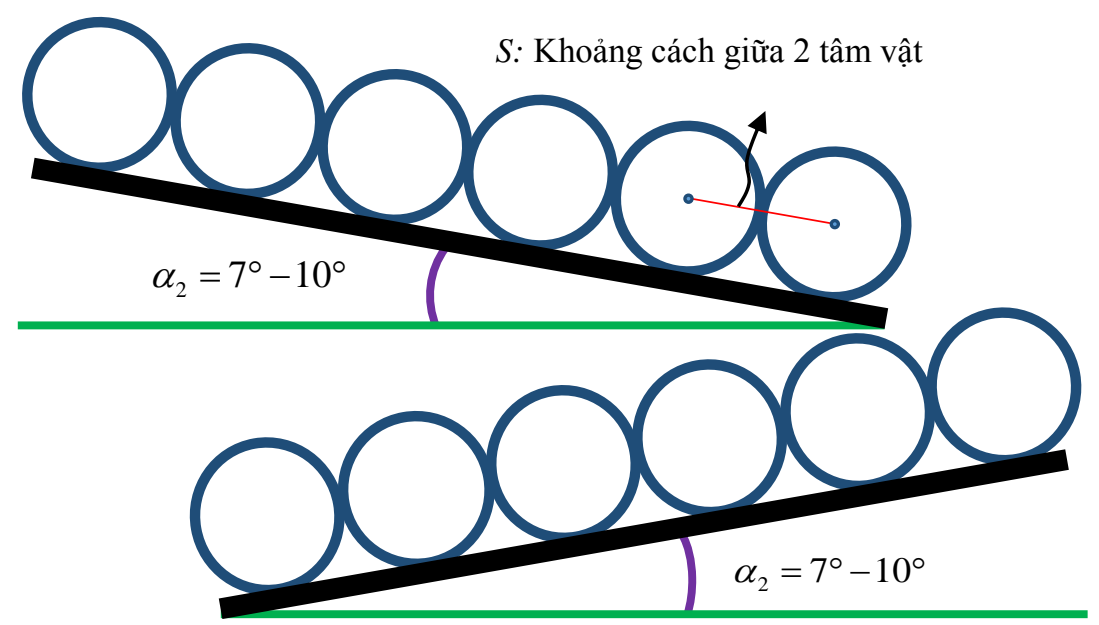

Hình 12: Góc nghiêng bộ phận thoát phôi

Do đó, tác giả đã tính toán và đưa ra góc nghiêng phù hợp với yêu cầu đặt ra là góc nghiêng $\alpha_{2}$ dao động trong khoảng $7^{\circ}-10^{\circ}$ (Hình 12), vì với góc nghiêng như vậy phôi sẽ đảm bảo được tốc độ thoát phôi nhanh và không bị nẩy hay va chạm làm lệch quỹ đạo di chuyển, đảm bảo đáp ứng được yêu cầu thời gian $t<4.2$ giây. Với góc nghiêng này thì vẫn chưa xảy ra hiện tượng vừa lăn vừa trượt. Tuy nhiên, nếu như yêu cầu đặt về mặt thời gian có sự thay đổi, ví dụ cần nhanh hơn nữa $(t<0.5$ giây), thì góc nghiêng sẽ có giá trị lớn hơn $\left(\alpha_{2} \geq 35^{\circ}\right)$ và có thể dẫn đến hiện tượng vừa lăn vừa trượt. Để đáp ứng được yêu cầu đặt ra của bài toán, thùng thoát phôi đã được thiết kế phân tầng, thành 2 tầng khác nhau và trên tấm dẫn hướng 
cho phôi sẽ có các gờ nổi ở 2 bên, mục đích không cho bề mặt in trên chai tiếp xúc với mặt phẳng của tấm dẫn hướng, từ đó sẽ đảm bảo việc mực in trên chai không bị nhòe hay lem. Ngoài ra, việc thiết kế thùng thoát phôi thành 2 tầng khác nhau và có đế phía dưới còn nhằm mục đích để người công nhân không phải luôn quan sát chai rớt ra mỗi khi chai in xong. Việc thiết kế thùng thoát phôi đa tầng sẽ giảm thiểu được tình trạng lem mực trên chai sau khi in cũng như tiết kiệm được diện tích thùng chứa phôi.

\section{KÊT QUẢ CHẾ TẢO VÀ THẢO LUẦN}

Sau khi tính toán và kiểm nghiệm trên phẩn mềm mô phỏng, tác giả đã chế tạo thành công máng cấp và thoát phôi theo đúng với thiết kế ban đầu (Hình 13). Kết quả kiểm nghiệm đối với máng cấp và thoát phôi hoàn toàn thỏa mãn yêu cầu đặt ra của bài toán, sai số đo đạc giữa kiểm nghiệm thực tế so với mô phỏng dưới $10 \%$, hoàn toàn có thể chấp nhận được. Vì trong thực tế mô hình vẫn còn bị ảnh hưởng bởi nhiều yếu tố khác như gió, rung động bởi môi trường xung quanh.

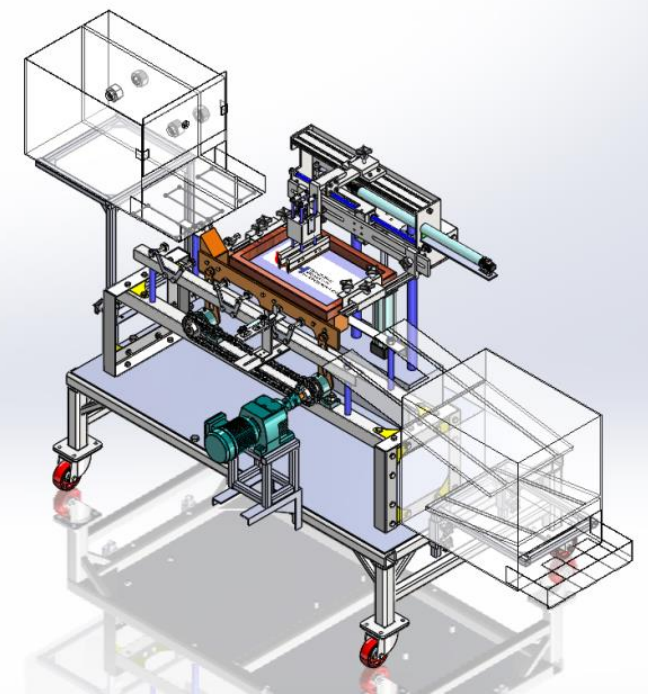

(a)

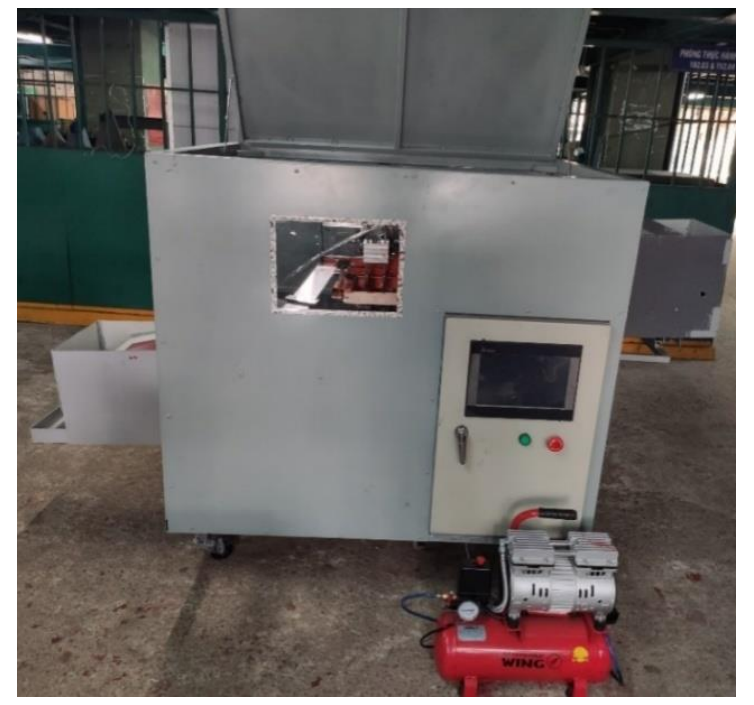

(b)

Hình 13: Hình ảnh máy theo thiết kế (a) và máy được chế tạo (b)

Thông qua các mô hình mô phỏng trên phần mềm Recurdyn và kết quả thu được lúc chế tạo thực nghiệm, tác giả đã đưa ra được những nhận xét như sau:

Góc nghiêng của thùng cấp phôi càng lớn thì càng tốn không gian của thùng chứa phôi. Tuy nhiên vật sẽ lăn càng nhanh, mà càng nhanh nó càng khiến cho vật bị bấp bênh khi vật va chạm vào khối đỡ chữ $V$ (vị trí chờ để chuyển phôi $\mathrm{V}$ - Hình 11). Trong bài toán này góc nghiêng $\alpha_{1}$ của thùng cấp phôi phải nằm trong khoảng $\left[0.14^{\circ} ; 2.3^{\circ}\right]$, để sao cho vật lăn vừa kịp vào vị trí chờ, đồng thời để giảm thiểu tối đa được lực va chạm với khối $\mathrm{V}$ (Hình 11).

Cũng trong bài toán này góc nghiêng $\alpha_{2}$ của thùng thoát phôi phải nằm trong khoảng $\left[7^{\circ} ; 10^{\circ}\right]$ để tốc độ thoát phôi đáp ứng được thời gian làm khô mực trên chai. Để có thể làm được việc đó, thùng thoát phôi cần được thiêt kế phân tầng. Từ có thể thu gọn kích thước máy cũng như đáp ứng được thời gian yêu cầu của bài toán.

Quãng đường vật đi càng xa, thì sai số của mô phỏng so với công thức tính toán lý thuyết (3) càng lớn. Vì trong mô phỏng cũng như trong môi trường thực tế, vật di chuyển sẽ gặp nhiều ảnh hưởng đến từ những yếu tố khác nhau. Từ đó quỹ đạo di chuyển của vật sẽ không còn là một đường thẳng nữa, mà có thể là các quỹ đạo cong khác nhau (Hình 14).

Như vậy kết quả mô phỏng so với công thức lý thuyết còn nhiều sai lệch, do trong môi trường mô phỏng, phần mềm Recurdyn đã xét đến các yếu tố khác như vật liệu và hình dạng của nắp và chai, độ cứng, độ giảm chấn, v.v... của từng vật liệu. Tuy nhiên kết quả mô phỏng ứng dụng vào phần thiết kế và chế tạo máy đã cho thấy máy làm việc đúng như yêu cầu đã đề ra. 


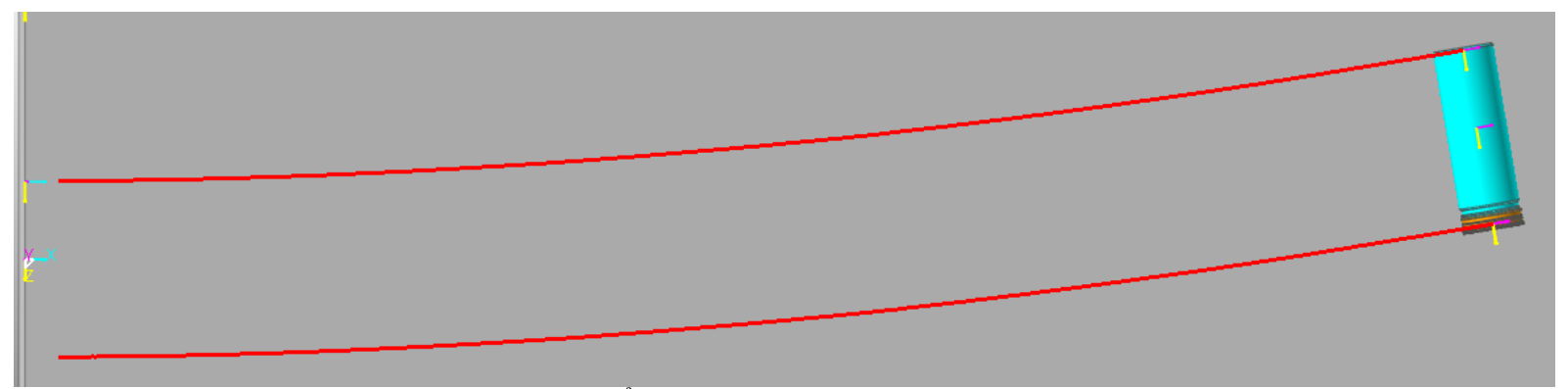

Hình 14: Quỹ đạo di chuyển của chai trong môi trường mô phỏng Recurdyn

\section{KẾT LUẦN}

Bài báo trình bày về việc tính toán, thiết kế và mô phỏng hệ thống cấp - thoát phôi tự động trong máy in lụa bán tự động kiểu mới. Các tác giả đã đưa ra được công thức tính toán lý thuyết vật lăn với ma sát, quy trình mô phỏng hiện tượng này trên phần mềm Recurdyn, dựa vào đó để thiết kế và chế tạo thành công máy in lụa bán tự động kiểu mới. Phương pháp và quy trình mô phỏng vật lăn trong Recurdyn này có thể được ứng dụng để mô phỏng các hiện tượng lăn phức tạp khác như vật có hình dạng bất kỳ hoặc vật vừa lăn vừa biến dạng, v.v... Từ đó, người dùng có thể hiểu rõ hơn về lợi ích của việc tính toán và mô phỏng trên phần mềm để có thể giảm thiểu được tối đa chi phí thiết kế, chế tạo cũng như rút ngắn được thời gian để tạo ra một sản phẩm cơ khí hoàn chỉnh.

\section{TÀI LIỆU THAM KHẢO}

[1] Siegfried Edels, Kensington, London, Arthur William Excell, Thornton, England. Silk Screen Printing Machines.United States Patent Office № 3,026,003. 20/03/1962.

[2] Louis Giuhert Dubult. Silk- Screen Printing Device.United States Patent Office № 3,113,510. 03/1962.

[3] Bessie Webber Fiegel, Bachelor of Science "Oklahoma College for WomenChickasha, Oklahoma. The origin and development of silk screen printing and its application as a homecraft, 08/1965.

[4] Peter Zimmer. Rotary Screen Printing Machine.United States Patent Office № 3,565,001. 02/1968.

[5] Lã Xuân Trường và các cộng sự. Nghiên cứu, thiết kế và chế tạo thử nghiệm máy in lụa tự động kiểu mới ứng dụng cho các cơ sở sản xuất vừa và nhỏ ở Việt Nam, luận văn đại học. Đại học Công nghiệp Tp.HCM, 2020.

[6] Zhiming Zhang, Jun Sun, Binbin Lu, Yaoshuai Duan. Turning mechanism and optimisation design of automatic screen printing machine. International Journal of Wireless and Mobile Computing, 2018 Vol.15 No.2, Pp.151 - 156.

[7] Hoang Minh Dang, Thanh Kiet Vo, Hung Linh Ao, Van Binh Phung, Nguyen Viet Duc. Design and development of the silkscreen printer with an innovative automatic mechanism of feeding and transporting workpieces. Journal of Mechanical Engineering Research \& Developments, Vol. 44, No. 1, 2021, Pp. 304-316.

[8] C. Viorica, C. Jana, E. Balan, and C. Mohora, the study of the screen printing quality depending on the surface to be printed. MATEC Web of Conferences, 2018, Vol.178, Pp. 1-6.

[9] Li Pengfei, Nie Luhua, Wang Bo and Li Jiakun, Research on rotary screen printing machine multi-axis motion control system based on CAN Bus. 2010 2nd International Conference on Advanced Computer Control, Shenyang, China, 2010, Pp. 39-42.

[10] S.A. Kacharen, N.N. Narwade, V.U. Mandle, H.V. Shiraskar, V.P. Sawant. Pneumatic Multicolour Screen Printing Machine, International Journal for Scientific Research \& Development Vol. 4, Issue 03, 2016, Pp. 1031-1033.

[11] G.R. Selase, V. Divine, D. Elorm, A. Joseph, A.F Emefa, A. Joana. Portable T-Shirt Printing Machine, Arts and Design Studies Vol.57, 2017, Pp. 36-51.

[12] Priyadharson, A. \& Surarapu, P.K. PLC-HMI based automatic screen printing system. International Journal of Mechanical Engineering and Technology, 2017, Pp.101-107. 
96 TÍNH TOÁN VÀ MÔ PHỎNG HIỆN TƯợNG MA SÁT TRƯợT ĐỐI VỚI VẬT LĂN ÚNG DỤNG TRONG THIÊT KÊ HỆ THỐNG CÂP - THOÁT PHÔI CỦA MÁY IN LƯA BÁN TỰ ĐỘNG KIỂU MỚI

[13] WANG Zhi-wei, MEI Shun-qi, DU Xing, LIU Ping, LIU Wei. Automatic Screen Printing Machine Control System Based on PLC. Light Industry Machinery, 2011, Pp. 53-55.

[14] N. Pandrea and D. Popa. Classical and Modern Approaches in the Theory of Mechanisms, John Wiley and Sons, 2017.

[15]M. J. Rider. Design and analysis of mechanisms: a planar approach, John Wiley \& Sons, Ltd, 2015.

[16] M. Sh. Sichani, On Efficient Modelling of Wheel-Rail Contact in Vehicle Dynamics Simulation. Doctoral Thesis, Stockholm, Sweden 2016, p. 121.

[17] V. L. Popov, Contact Mechanics and Friction: Physical Principles and Applications, 2nd ed. 2017 ed. Springer, 2017.

[18]J. J. K. e. Bo Jacobson, Rolling Contact Phenomena, 1 ed. (no. International Centre for Mechanical Sciences 411). Springer-Verlag Wien, 2000.

[19] H.M. Dang, V.P. Bui, V.B. Phung, S.S. Gavriushin, V.D. Nguyen, PAMMS - Procedure for Automation of Mathematical Modeling and Solution of Mechanical system: Application for the Design of an Innovative Fruit and Vegetable Washer. Journal of Mechanical Engineering Research and Developments, vol. 43, no. 3, 2020, Pp. 429442.

[20] G. Dieter and L. Schmidt. Engineering Design, 5th Edition, McGraw-Hill Higher Education, 2012.

[21] A. Ruina and R. Pratap, Introduction to Statics and Dynamics. 2001, p. 626.

[22] Nobuyuki Shimizu, RecurDyn for Beginners - Innovation for Design \& Analysis with Multibody Dynamics. FunctionBay, Inc. 2015, p.295

[23] https://support.functionbay.com/. (Ngày kiểm tra: 18/03/2021)

[24] B. N. J. Persson, Theory and simulation of sliding friction, Physical Review Letters, 08/23/1993, vol. 71, no. 8, pp. 1212-1215.

[25] M. R. So/rensen, K. W. Jacobsen, and P. Stoltze, Simulations of atomic-scale sliding friction, Physical Review B, 01/15/ 1996, vol. 53, no. 4, pp. 2101-2113. 